\title{
Reconstruction of leaking gastric pouch after redo Rouxen-Y gastric bypass - revisionary surgery strategy
}

\author{
Wojciech K. Karcz ${ }^{1}$, Cheng Zhou², William Braun ${ }^{3}$, Piotr Małczak², Ulrich Wellner ${ }^{1}$ \\ ${ }^{1}$ Clinic for Surgery, Department of Metabolic and Obesity Surgery, Schleswig-Holstein University Clinic, Campus Lübeck, Germany \\ ${ }^{2}$ Department of Hepatobiliary Surgery, Wuhan No. 1 Hospital, Wuhan, China \\ ${ }^{3}$ Department of Upper GI and Bariatric Surgery, Metro North Hospital and Health Service, Brisbane, QLD, Australia \\ ${ }^{4} 2^{\text {nd }}$ Department of General Surgery, Jagiellonian University Medical College, Krakow, Poland
}

Videosurgery Miniinv 2016; 11 (1): 9-13 DOI: $10.5114 /$ wiitm.2016.57485

\begin{abstract}
With the rapid development of the knowledge and techniques in bariatric surgery, as well as extensive expansion of indications for metabolic operations, surgeons have started to realize that the procedures they choose are not always suited to the needs and behavior of patients. Second and very frequently third revisional procedures are thus necessary in order to achieve desired outcomes and fulfill patients' expectations. Restoration of restriction is commonly the first procedure, but not all may benefit from it. We hence want to illustrate the decision-making process by presenting a patient with the background of initial Rouxen-Y gastric bypass (RYGB) and redo-RYGB, who then underwent a further revision by a 2-stage approach due to weight regain. The first stage involved shortening of the common channel and the second one reconstruction of his gastric pouch with duodenal switch (DS). Consequently we wish to focus here on a discussion about operative techniques and strategies to improve outcomes in such patients.
\end{abstract}

Key words: bariatric surgery, restriction, revisionary surgery, malabsorption.

\section{Introduction}

The nature of the operative techniques and their complexity in some cases make early or late complications of bariatric surgery inevitable. Insufficient weight loss or weight regain, which is classified as a late complication, are the most common indications for revisional operations [1-4]. According to the literature, weight regain in the vicinity of $40 \%$ following a gastric bypass procedure (GBP) is common. On the other hand, the rate of revisional operations following biliopancreatic diversion (BPD) was lower than GBP $[5,6]$. Since there are no formal data available to us to date, the role of gastric pouch or gastroenterostomy dilation as a cause for these late failures is not clear. However, the overall mortality after re-operative cases is reported to be as high as $56 \%$ in some series. This means that bariatric revisional procedures are technically more demanding and associated with potentially poorer outcomes in some instances. Restoration of restriction by reduction of the gastric pouch in the GBP, suture plication of a dilated gastric pouch with or without endoscopy, implantation of a fobi-ring (GaBP) or Minimizer ring [7-9], and similar techniques (Bessler) [10] are all part of our armamentarium in order to achieve the restriction we need. Nonetheless, there is a paucity of data demonstrating that revisional surgery through restriction is effective in every case, and on the contrary we all know that in some situations even the highest degree of restriction does not result in a desired outcome at all.

\section{Address for correspondence}

Assoc. Prof. Dr. med. Cheng Zhou, Department Hepatobiliary Surgery, Wuhan No. 1 Hospital, No. 215 ZhongShan Road, QiaoKou District, Wuhan, Hubei Province, 430022 China, e-mail: cheng.zhou@whyyy.com 


\section{Aim}

The aim of our study is to present our experience in treating patients with failed primary bariatric procedures and provide some insight into the methods we use at our clinic.

\section{Material and methods}

A 38-year-old male patient was reviewed in our bariatric outpatient clinic presenting with weight regain after Rouxen-Y gastric bypass (RGBP) (Figure 1) and its subsequent revision (Figure 2 ). Body mass index (BMI) of $52 \mathrm{~kg} / \mathrm{m}^{2}$ with no comorbidities was the indication for the initial RYGB. Having experienced weight regain he underwent the "redo" gastric bypass, which was focused on gastric pouch volume reduction. After this "redo" procedure, his body weight continued to increase and reached $155 \mathrm{~kg}$. We did not have any other information from different centers he had been previously treated at. We went on and performed our routine preoperative investigations including 3D CT reconstruction volumetry and upper gastro-intestinal endoscopy. Through these investigations it was established that during his "redo" operation the gastric pouch was almost completely obliterated and the alimentary limb was in fact anastomosed directly onto the esophagus at the level of the gastro-esophageal junction, and no stomach at all could be visualized on the endoscopy. The patient's nutritional and psychological status was also thoroughly evaluated. Following numerous discussions, he agreed to undergo a further surgical procedure. Based on this patient's bodyweight, BMI, and especially his intra-abdominal fat status, a twostage operative approach was adopted. The video with the surgical technique is attached.

\section{Results}

First stage manual: distal alimentary limb re-anastomosis. The procedure is known as the Lemmens-Himpens operation (Figure 3)

After laparoscopic ports were placed under direct vision, the peritoneal cavity was inspected. The previous proximal anastomosis was exposed and examined, and no gastric pouch was detected. The distance from the proximal presumably gastro-jejunal anastomosis (GJA) to the jejunal-jejunal anas-

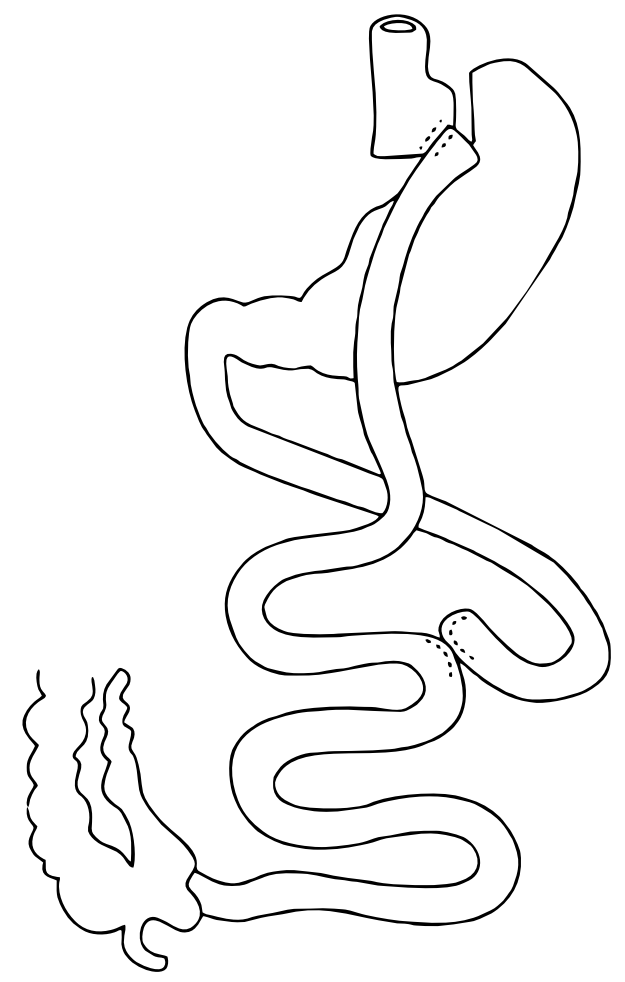

Figure 1. Gastric bypass procedure (RYGB)

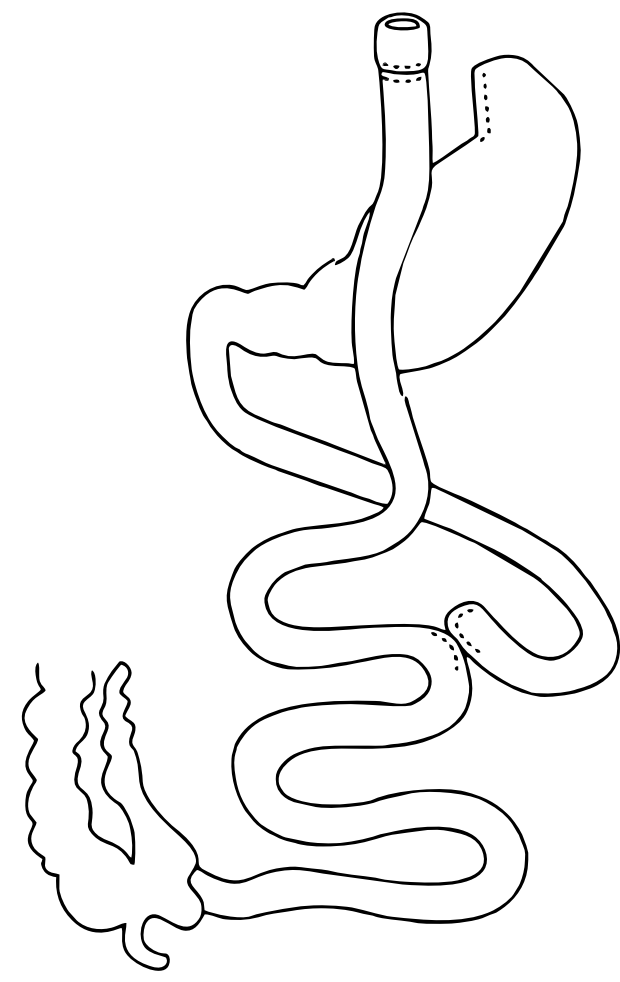

Figure 2. Redo gastric bypass procedure (by endoscopy - no gastric pouch) 
tomosis (JJA) and from the Treitz ligament to the jejuno-jejunal anastomosis was measured with an atraumatic intestinal grasper, and was $150 \mathrm{~cm}$ and $50 \mathrm{~cm}$ respectively. Following this the appendix was identified in the right lower quadrant and removed by a linear stapler with a white cartridge at the base. The common channel was then measured and was approximately $450 \mathrm{~cm}$ long. A marking suture was then placed at a spot approximately $150 \mathrm{~cm}$ proximal to the ileo-cecal junction, which was followed by a proximal holding suture with Vicryl (3/0) (Johnson \& Johnson, Ethicon).

Following that, the jejunal continuity was re-established, and the alimentary limb was transected immediately before it entered the new anastomosis by a linear stapling device (GIA White, $60 \mathrm{~mm}$, Covidien, Dublin, Ireland). The distal end of the original alimentary limb was brought to the fore-marked ileum and the side-to-side anastomosis was performed (GIA White, 60 mm: V-loc (90) 3/0, Suture, Covidien, Dublin, Ireland). For internal hernia prevention, closure of the mesentery was carried out. The first stage of the procedure was thus completed and a drain was placed.

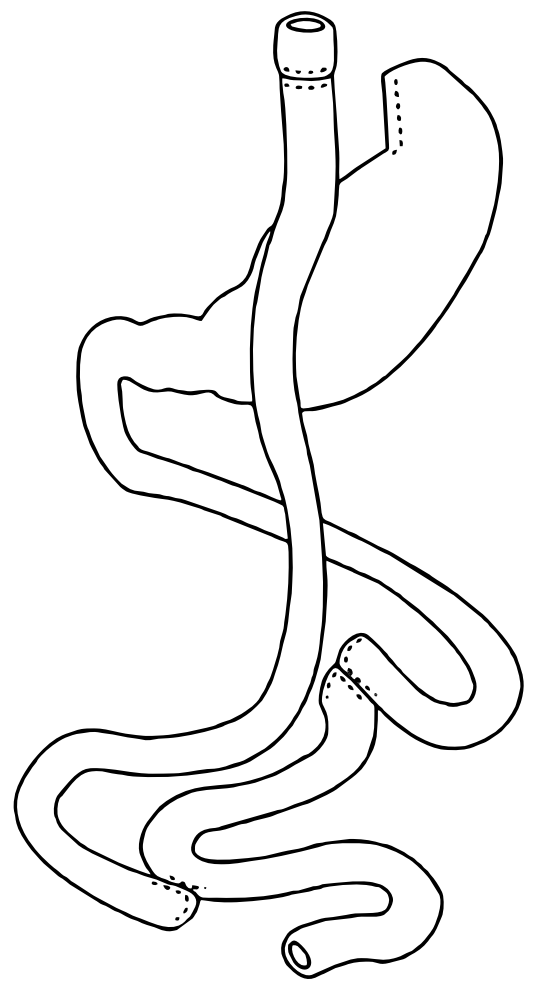

Figure 3. Lemmens-Himpens revisionary operation

\section{Metabolic and bariatric results after first stage}

The patient underwent the follow-up program with the assessments of his nutritional status and his level of compliance in taking supplements. He had at that stage lost $41 \mathrm{~kg}$ in 6 months without any signs of malnutrition on his pathology testing. It was exactly the desired clinical effect we wanted to achieve. There was no side effect or complication following this Lemmens-Himpens operation, and consequently we decided to proceed with the second stage of the procedure, which would involve gastric pouch reconstruction with a duodenal switch (Figure 4).

\section{Second stage manual: reconstruction of stomach pouch with duodenal switch of the alimentary limb (Figure 4)}

As expected, remarkable reduction in the amount of visceral fat was evident during the abdominal exploration at the beginning of the second stage of our operation. After oral introduction of a $32 \mathrm{Fr}$ bougie the jejunal alimentary limb was transected $5 \mathrm{~cm}$

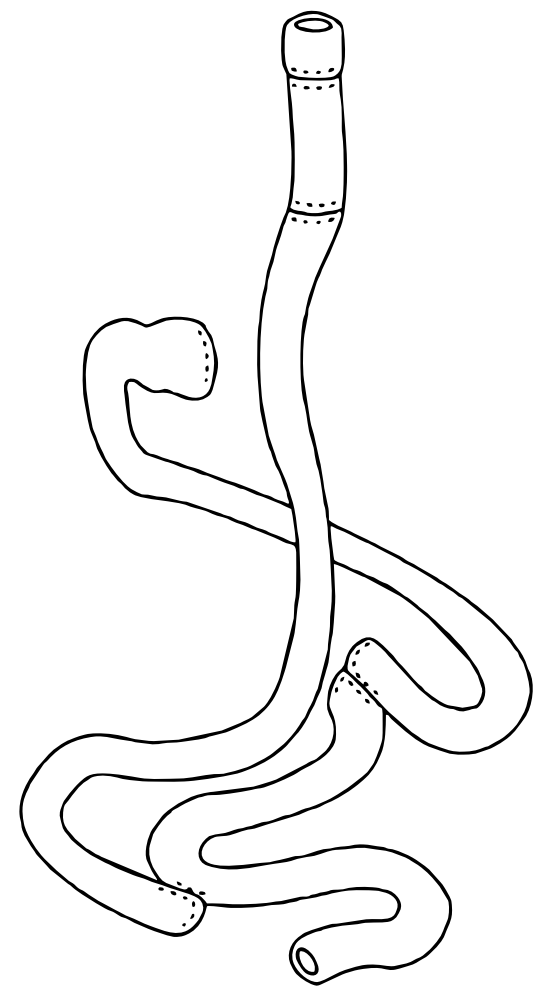

Figure 4. Gastric pouch reconstruction with DS and duodeno-jejunostomy 
distal to the previous "gastro"-jejunal anastomosis with a linear stapler (GIA White, $60 \mathrm{~mm}$, Covidien, Dublin, Ireland). Following this, careful adhesiolysis and dissection of all the components of the upper alimentary tract was performed, exposing both diaphragmatic crura, until the lower part of the esophagus and the gastro-esophageal junction were on view. We then placed a PDS 2/0 purse string suture into the new pouch, designed for securing the anvil of a circular stapler for a subsequent anastomosis. LigaSure V (Covidien, Dublin, Ireland) was used for dissection along the greater and lesser curvature of the remnant stomach. A longitudinal gastrostomy was made along the greater curvature using a diathermy hook to accommodate the circular anastomotic stapler (CEA 25 mm, Covidien, Dublin, Ireland). Once the gastric pouch and the remnant stomach were well positioned, we re-established gastric continuity. The anastomosis was then re-enforced with hand-sewn sutures (V-loc 3/0, 90, Covidien, Dublin, Ireland). A wide sleeve gastrectomy was then performed using a linear stapling device (GIA Blue, $60 \mathrm{~mm}$, Covidien, Dublin, Ireland).

The duodenum was then separated and transected with GIA (GIA White, $60 \mathrm{~mm}$, Covidien, Dublin, Ireland). A hand-sewn end-to-side anastomosis was then fashioned between the post-pyloric duodenum and jejunal alimentary limb with V-loc (90) 3/0 (Covidien, Dublin, Ireland). Two drainage tubes were placed bilaterally as the final step at the end of the procedure.

\section{Metabolic and bariatric results after second stage}

In the regular follow-up program after the second stage procedure we did not notice any signs of malabsorption or nutritional problems. The patient lost a further $21 \mathrm{~kg}$, and his body weight was $81 \mathrm{~kg}$ a year later.

\section{Discussion}

Although a gastric bypass procedure still remains the gold standard for bariatric surgery, it shows up to $40 \%$ weight regain during the long-term follow-up. Chistou [11] reported that about 35\% of GBP patients have BMI of greater than 35 based on long-term observation. A wide gastroenterostomy, larger pouch and return to poor eating habits remain the main reasons for the failure in patients undergoing gastric bypass. As a rule, restoration of restriction is considered to be the first revisionary choice after gastric bypass. Müller et al. showed that gastric pouch dilation (greater than $30 \mathrm{ml}$ ) was associated with bodyweight regain and metabolic disturbances and could be corrected by restoration of restriction [12-14]. In our case there was no room for a further restrictive procedure as there was no pouch present that could have been made smaller. We diagnosed it with preoperative 3D $\mathrm{CT}$ volumetry and endoscopy even before the laparoscopic exploration. As we all know, choosing a malabsorptive procedure is not an easy decision, and we had no other option. Based on the indications, such as $\mathrm{BMI}>40 \mathrm{~kg} / \mathrm{m}^{2}$, bodyweight regain, and most importantly visceral fat accumulation, a two-stage operation was planned and carried out. Patient's age and his personal preference had also helped in our decision to adopt this approach. The option to perform the whole procedure during one operation would have been associated with much higher overall risk. Furthermore, the first stage of our approach allowed us to inspect the stomach and the alimentary limb and ascertain whether the second stage could be at all feasible. After the first stage the patient lost more weight than we had expected, so that BMI during the second step was already under 40. During the $2^{\text {nd }}$ stage, the residual gastric pouch was refashioned, and duodenal switch was performed. The operation resulted in introduction of a further $80 \mathrm{ml}$ of stomach into the alimentary channel, improving the sense of satiety for this patient. It also diminished the early dumping syndrome and slowed down the normally rapid esophago-jejunal food passage. It is worth mentioning that the length of the common channel (CC) is one of the decisive factors for the result of a revisional malabsorptive operation. Normally, the CC is set at 75-100 cm [15-17], but after GBP we believe that it should not be shorter than $150 \mathrm{~cm}$.

\section{Conclusions}

Introduction of malabsorption in addition to restriction ought to be considered for selected patients. These choices must be based on thorough understanding of pathologic processes in metabolic disorders and careful consideration of individual needs.

\section{Conflict of interest}

The authors declare no conflict of interest. 


\section{References}

1. Gawdat K. Bariatric re-operations: are they preventable? Obes Surg 2000; 10: 525-9.

2. Gagner M, Gentileschi P, de Csepel J, et al. Laparoscopic reoperative bariatric surgery: experience from 27 consecutive patients. Obes Surg 2002; 12: 254-60.

3. Kuesters S, Grueneberger JM, Baumann T, et al. Revisionary bariatric surgery: indications and outcome of 100 consecutive operations at a single center. Surg Endosc 2012; 26: 1718-23.

4. Calmes JM, Giusti V, Suter M. Reoperative laparoscopic Rouxen-Y gastric bypass: an experience with 49 cases. Obes Surg 2005; 15: 316-22.

5. van Gemert WG, van Wersch MM, Greve JW, Soeters PB. Revisional surgery after failed vertical banded gastroplasty: restoration of vertical banded gastroplasty or conversion to gastric bypass. Obes Surg 1998; 8: 21-8.

6. Kuesters S, Marjanovic G, Karcz WK. Redo operations after bariatric and metabolic surgery. Zentralbl Chir 2009; 134: 50-6.

7. Fobi MA, Lee $\mathrm{H}$. The surgical technique of the Fobi-Pouch operation for obesity (the transected silastic vertical gastric bypass). Obes Surg 1998; 8: 283-8.

8. Fobi MA, Lee H, Felahy B, et al. Choosing an operation for weight control, and the transected banded gastric bypass. Obes Surg 2005; 15: 114-21.

9. Karcz WK, Suslin D, Baumann T, et al. To have or not to have the ring: early and late surgical complications after banded Rouxen-Y gastric bypass. Videosurgery Miniinv 2008; 3: 53-65.

10. Bessler M, Daud A, Kim T, DiGiorgi M. Prospective randomized trial of banded versus nonbanded gastric bypass for the super obese: early results. Surg Obes Relat Dis 2007; 3: 480-4.

11. Christou NV, Look D, Maclean LD. Weight gain after short- and long-limb gastric bypass in patients followed for longer than 10 years. Ann Surg 2006; 244: 734-40.

12. Langer FB, Bohdjalian A, Felberbauer FX, et al. Does gastric dilatation limit the success of sleeve gastrectomy as a sole operation for morbid obesity? Obes Surg 2006; 16: 166-71.

13. Muller MK, Wildi S, Scholz T, et al. Laparoscopic pouch resizing and redo of gastro-jejunal anastomosis for pouch dilatation following gastric bypass. Obes Surg 2005; 15: 1089-95.

14. Karcz WK, Kuesters S, Marjanovic G, et al. 3D-MSCT gastric pouch volumetry in bariatric surgery - preliminary clinical results. Obes Surg 2009; 19: 508-16.

15. Hess DS, Hess DW, Oakley RS. The biliopancreatic diversion with the duodenal switch: results beyond 10 years. Obes Surg 2005; 15: 408-16.

16. Marceau P, Hould FS, Potvin M, et al. Biliopancreatic diversion (doudenal switch procedure). Eur J Gastroenterol Hepatol 1999. 11: 99-103.

17. Karcz WK, Kuesters S, Marjanovic G, Grueneberger J. Duodeno-entero omega switch - more physiological technique in metabolic surgery. Videosurgery Miniinv 2013; 8: 273-9.

Received: 1.09.2015, accepted: 14.12.2015. 